\title{
Programa de fortalecimiento de la Cultura Organizacional en los docentes de la Universidad Adventista de Bolivia, gestión 1/2020
}

\author{
Program for strengthening the Organizational Culture in the teachers of the \\ Adventist University of Bolivia, management 1/2020
}

Alicia Camacho Camacho
alicia.camacho@uab.edu.bo
Código ORCID: 0000-0002-8132-4693
Universidad Adventista de Bolivia, Cochabamba, Bolivia

| Artículo recibido en octubre 2020

I Arbitrado en noviembre 2020

| Aceptado en diciembre 2020

I Publicado en enero 2021

\section{RESUMEN}

Palabras clave:

Cultura organizacional, docentes

universitarios, percepción docente, fortalecimiento organizacional
El comportamiento de una institución depende en gran medida de cómo está siendo aceptada la cultura organizacional en su personal de trabajo. La presente investigación tuvo como objetivo caracterizar la percepción de los docentes sobre la cultura organizacional de la Universidad Adventista de Bolivia durante la gestión 1/2020. Este estudio se apoyó para su construcción teórico metodológica entre otros autores por Schein (1988) Chiavenato (2007) White (1975) Llanos (2016) y Denison (1990) El método se sustentó en el paradigma positivista bajo el diseño no experimental con un enfoque cuantitativo de tipo descriptivo, empleando la técnica entrevista semiestructurada. La mayoría de los docentes perciben a la universidad con una cultura organizacional fuerte pero que no es el ideal. Existen deficiencias que se encuentran evitando que la cultura sea totalmente apropiada por el docente. Por lo que se recomienda implicar al personal laboral para la ejecución de programas de inducción laboral y al conocimiento de la misión institucional.

\section{ABSTRACT}

The behavior of an institution depends to a large extent on how the organizational culture is being accepted in its workforce. The present research aimed to characterize the teachers' perception of the organizational culture of the Adventist University of Bolivia during the 1/2020 administration. This study was supported for its theoretical-methodological construction among other authors by Schein (1988) Chiavenato (2007) White (1975) Llanos (2016) and Denison (1990) The method was based on the positivist paradigm under the non-experimental design with a descriptive quantitative approach, using the semi-structured interview technique. Most teachers perceive the university as having a strong organizational culture, but it is not ideal. There are deficiencies that are preventing the culture from being fully appropriate by the teacher. Therefore, it is recommended to involve the workforce for the execution of job induction programs and knowledge of the institutional mission. 


\section{INTRODUCCIÓN}

La cultura es aplicada a diversas unidades sociales que hayan podido aprender y establecer una visión de sí mismas y del medio que las rodea. En el primer nivel se encuentran las civilizaciones y culturas permanentes del mundo occidental y oriental, seguido por los países que tienen identidad étnica y dentro de las mismas tienen grupos étnicos con culturas diversas (Schein, 1988) Cada uno ha desarrollado su propia forma de percibir el mundo externo. Del mismo modo ocurre con el nivel de ocupación, profesión o comunidad laboral, lo cual fundamentó el análisis de la presente investigación que tuvo como objetivo: Caracterizar la percepción de los docentes sobre la cultura organizacional de la Universidad Adventista de Bolivia durante la gestión 1/2020. La cual se encuentra en el municipio de Vinto, cuarta sección municipal de la provincia de Quillacollo, perteneciente al departamento de Cochabamba del Estado Plurinacional de Bolivia (Amdeco, 2018).

Menciona Chiavenato (2007) que "cada organización tiene su cultura organizacional o cultura corporativa. Para conocer una organización, el primer paso es conocer la cultura de esta. Formar parte de una organización significa asimilar su cultura" (p. 83). Todas las organizaciones tienen un modo característico en el que las personas interactúan y este aspecto debe ser debidamente ordenado, es decir, que la organización conozca a dónde se dirige y que el trabajador asimile este aspecto y trabaje en conjunto con esa perspectiva.

El comportamiento ideal de una institución depende de cómo está siendo aceptada su cultura organizacional. Esta sólo se puede observar en los efectos y consecuencias que puedan impactar sobre el personal de trabajo.

Como la cultura de una organización rige el comportamiento de cada individuo perteneciente, es necesario tener el debido cuidado al momento de introducir la normativa, valores y principios al nuevo trabajador y al que ya se encuentra trabajando en la institución. El modo de expresar y de aprehender estos asuntos será de relevancia para que exista una identificación y compromiso adecuados con la institución.

En la actualidad diversas organizaciones se encuentran constantemente preocupadas por cómo incentivar el mejor desempeño de sus trabajadores. Dichas recurren a estrategias externas que les ayuden a tener mejor productividad laboral, sin darse cuenta que es necesario primero examinar cómo está siendo percibida la cultura organizacional por parte del personal de trabajo. La cultura es la base de cómo es la organización, si esta es abierta a sus empleados o si se encuentra cerrada a las nuevas oportunidades. No por nada menciona Chiavenato (2009) que:

Cada organización tiene características distintivas, como su estilo de vida, comportamiento, mentalidad, presencia y personalidad. Además, cada una tiene rasgos distintivos que no son tangibles o mensurables. Muchos fenómenos que ocurren en una organización se derivan de su cultura, que es como su código genético. (p.119)

Para que estos efectos y consecuencias puedan ser positivos se debe tener una buena alineación de objetivos y metas, las cuales están trazadas por la institución. Dependerá también si la organización tiene una cultura conservadora o adaptable. Las conservadoras, menciona Chiavenato (2009) son las que se caracterizan porque adoptan y preservan ideas, valores, costumbres y tradiciones que no cambian con el tiempo, a pesar de las transformaciones del entorno, son rígidas y conservadoras, puede ser también que su estructura de liderazgo sea vertical.

Las adaptables son muy flexibles a los cambios y se encuentran en revisión constante 
de su cultura, pero a la vez pueden tener algunas dificultades, como la pérdida de identidad por los diversos cambios que tiene dentro de su organización y la cual obviamente afecta también al trabajador. Este tipo de cultura está caracterizada por su maleabilidad y flexibilidad.

Es necesario tener cambios para el logro de objetivos, pero también se debe tener un grado de estabilidad, especialmente en los fundamentos que caracterizan a la institución, como son los valores, la misión y la filosofía en la cual se encuentran enmarcados. La misión es: Somos una institución de Educación Superior comprometida con la formación integral de profesionales competentes, que promuevan el desarrollo de ciencia y tecnología y practiquen el servicio a Dios y a la sociedad, fundamentados en la cosmovisión cristiana que sustenta la Iglesia Adventista del s.n. Séptimo Día (UAB, 2018, s.n.)

En el caso que ocupó este estudio el ser humano es considerado de forma integral, siendo así es necesario el crecimiento físico, mental, social y espiritual. Al respecto dice White (1975) "La verdadera educación es una ciencia grandiosa, porque se funda en el temor del Señor, que es el principio de la sabiduría" (p. 41) es fundamental el temor a Dios y su palabra porque este es el que direcciona todo:

Cristo es el más grande Maestro que este mundo haya conocido; y no es del agrado del Señor Jesús que los súbditos de su reino, por los cuales murió, sean educados de manera tal que sean inducidos a colocar la sabiduría de los hombres en primera línea y releguen la sabiduría de Dios, según se revela en su Santa Palabra, a la última fila. (White,1975, p. 41)

En Proverbios 2:6-7 se registra lo siguiente "Porque Jehová da la sabiduría, y de su boca viene el conocimiento y la inteligencia. El provee de sana sabiduría a los rectos; Es escudo a los que caminan rectamente." (Reina y Valera, 1960). Dios es el dueño de nuestro saber y el conocimiento que cada ser humano puede adquirir, bajo su voluntad esta la ciencia y todo lo que hay en la tierra.

Entonces la educación y toda la ciencia debe estar basado no solo para la vida en esta tierra si no más allá de eso, como lo menciona White (1975). "La verdadera educación preparará a los niños y los jóvenes para la vida presente y la venidera, para una herencia en la patria mejor, es decir, la celestial" (p. 41). Es así que el conocimiento está bajo el orden divino de Dios y todo lo que se hace debe ser para fortalecer los hábitos buenos de la vida que preparen al ser humano para la eternidad.

Estos principios divinos rectores propendieron al conocimiento de la problemática vinculada a la Universidad Adventista de Bolivia, la cual está relacionada con el modo de percepción de los docentes hacia la cultura organizacional que esta promociona. La percepción de cada individuo es única y singular, la aceptación de la cultura organizacional dependerá de cómo es transmitida al docente. En este caso la percepción del docente viene a ser de manera general diferente en cada organización, así como lo menciona Llanos (2016):

Siendo que, a las culturas, no se
les puede dar un juicio de valor,
simplemente son diferentes, pero
por otro lado se debe considerar
que en la integración con los
diferentes grupos y frente a los
valores y principios universales
que regulan la convivencia entre
las personas, se debe buscar el
bienestar común. (p. 11)

Es justamente la integración al grupo que no se percibe que exista en la UAB, ya que a causa de que un porcentaje de docentes es 
de contrato de tiempo parcial, este se limita sólo a desempeñar la función laboral, al ser de tiempo parcial y perteneciente a niveles bajos, la administración no tiene suficientes canales de comunicación para llegar hasta ellos y poder unificar las ideas entre todos los docentes. Entonces la promoción de la cultura organizativa se maneja en los niveles altos e intermedios y no llega hasta los docentes, lo cual puede provocar la falta de compromiso hacia los propósitos que la institución persigue.

En la presente investigación indagó la percepción de los docentes sobre la cultura organizativa de la Universidad Adventista de Bolivia. La percepción individual de cada trabajador sobre la institución para la cual trabaja, es importante, ya que eso influirá en su desenvolvimiento dentro la organización y su desempeño será eficaz o deficiente dependiendo cómo acepte la cultura organizacional, lo cual es el conjunto de normas, valores, reglas, costumbres y todo lo relacionado a la institución. Los docentes de la universidad son la representación directa hacia los estudiantes quienes son los clientes principales de la institución, ahí radica la importancia de que esta población tenga claro lo qué la universidad busca alcanzar y cuál es el rol docente en ese proceso.

Por lo mencionado, dicha investigación ayudó a conocer de manera detallada bajo cuatro dimensiones (implicación, consistencia, adaptabilidad y misión) la percepción del docente sobre la cultura institucional y de ese modo crear estrategias de acción en la elaboración de un programa de fortalecimiento de cultura organizacional con el fin de que exista una mejor aceptación e identificación con la cultura organizativa de la institución. De allí que la premisa general se basó en responder la interrogante ¿Cuál es la percepción de los docentes, sobre las características de la cultura organizacional en la Universidad Adventista de Bolivia durante la gestión 2020?

\section{Perspectiva teórica}

\section{Definición de cultura organizacional}

Para definir cultura organizacional primero es necesario saber que es cultura, la cual puede aplicarse desde las expresiones del arte hasta las costumbres y valores de un grupo de personas o comunidad, es decir son características que mantiene una identidad propia. Chiavenato (2009) la define de la siguiente forma:

Cultura es un término general que se emplea con dos acepciones diferentes. Por una parte, significa el conjunto de costumbres y realizaciones de una época o de un pueblo y, por la otra, se refiere a las artes, la erudición y demás manifestaciones complejas del intelecto y la sensibilidad humana, consideradas en conjunto. (p. 176)

El término de cultura en las organizaciones es reciente a nivel mundial, antes de 1980 eran pocos los autores que se manifestaban sobre este tema, este ha ido evolucionando a lo largo del tiempo junto con las concepciones respecto a las relaciones laborales, esto se dio específicamente gracias la inclusión de la psicología en el mundo de las organizaciones empresariales, lo cual permite el estudio del comportamiento y las relaciones interpersonales dentro de la misma. Refiriéndose al rol del psicólogo, menciona Llanos (2016) lo siguiente:

Siendo las organizaciones, parte del sistema social y siendo que la psicología pone atención a toda la dinámica de las personas al interior de las mismas, se empieza a tener una concepción diferente. Se presta mayor atención al personal, ya no 
como recursos, sino como personas independientes que forman parte de un todo y traen consigo un bagaje; no solo de conocimientos, sino también de experiencias, y cuyas personalidades interactúan entre sí al interior de las organizaciones. (p. 12)

Es de ese modo que se va construyendo el concepto de cultura organizacional. Una de las primeras definiciones fue dada por Pettigrew (1979) citado por Llanos (2016) quien la describe como "el sistema de significados públicamente y colectivamente aceptados operando para un grupo determinado en un tiempo dado" (p. 12). La conceptualización fue en avance hasta que Edgar Schein en 1988, brinda una definición más amplia en su libro titulado La cultura empresarial y el liderazgo, que hasta ahora se utiliza como referencia primordial para hablar sobre cultura organizacional y en la cual también se basaron la mayoría de los autores actuales que estudian está temática. Por eso y otros aspectos, es considerado uno de los padres de la psicología de las organizaciones. La definición que brinda sobre la cultura es la siguiente:

Cultura es un modelo de presunciones básicas inventadas, descubiertas o desarrolladas por un grupo dado al ir aprendiendo a enfrentarse con sus problemas de adaptación externa e integración interna, que hayan ejercido la suficiente influencia para ser consideradas válidas y en consecuencia, ser enseñadas a los nuevos miembros como el modo correcto de percibir, pensar y sentir esos problemas. (p. 25)

Shein (1988) no menciona la conducta manifiesta lo cual vienen a ser las normas, valores, reglas, etc. Estás vienen determinadas por la predisposición de la cultura, su modo de sentir, percibir y pensar, como también por las contingencias o el modo de pensar que derivan del medio externo en el que los miembros se encuentran. Menciona también que para tener un concepto claro y amplio de este término se debe evitar lo siguiente:

Los análisis o interpretaciones erróneas dan lugar a cuatro tipo de engaños sobre la conceptualización de la cultura empresarial: que no se consigan entender las consecuencias dinámicas de los fenómenos culturales, que se ponga demasiado énfasis en el proceso de aprendizaje cultural (sociabilización) y escaso énfasis en el contenido de lo que verdaderamente se aprende (la verdadera cultura) que se confundan partes de la cultura son la totalidad cultural y que se confundan las manifestaciones superficiales de la cultura con el esquema subyacente, o con lo que podemos considerar la esencia o núcleo de la cultura. (p. 59)

Evidentemente las cuatro interpretaciones se encuentran entrelazadas entre sí y es relevante evitar la confusión de términos sino más bien, percibir desde diversos ángulos el todo de la cultura de una determinada institución y trabajar cada uno de esos aspectos con el objetivo de fortalecerlas conjuntamente. Entonces, en el estudio de las organizaciones, cultura se refiere a la forma de vida de la organización en todos sus aspectos, sus ideas, creencias, costumbres, reglas, técnicas, etc. En este sentido, todos los seres humanos estamos dotados de una cultura porque formamos parte de un sistema cultural (Chiavenato, 2009).

No existe organización sin una cultura inherente, que la identifique, la distinga y oriente su accionar y modos de hacer, rigiendo sus percepciones y la imagen que sus públicos tengan de ella (Rodríguez, 2004). De ese modo la definición conceptual 
de este término, fue en progreso. Chiavenato (2007) menciona:

La cultura organizacional representa las normas informales, no escritas, que orientan el comportamiento de los miembros de una organización en el día a día y que dirigen sus acciones en la realización de los objetivos organizacionales. Es el conjunto de hábitos y creencias establecidos por medio de normas, valores, actitudes y expectativas que comparten todos los miembros de la organización. (p. 84)

Es importante hacer hincapié en que la cultura es lo que se comparte de manera conjunta con todos los miembros de una organización, de ese modo estos miembros lleguen a identificarse $y$ apropiarse de la identidad organizacional. De acuerdo a investigaciones realizadas por G. Hofstade, B. Neuijen, D. Ohayv y G. Sanders (1991), citados por Llanos (2016) la cultura organizacional, ha sido definida por diez características básicas:

1. La identidad de los miembros: El grado en que los empleados se identifican con la organización como un todo y no solo con su tipo de trabajo o campo de conocimientos profesionales.

2. Énfasis en el grupo: el grado en que las actividades laborales se organizan en torno a grupo y no personas.

3. El enfoque hacia las personas: El grado en que las decisiones de la administración toman en cuenta las repercusiones que los resultados tendrán en los miembros de la organización.

4. La integración en unidades: El grado en que se fomenta que las unidades de la organización funcionen de forma coordinada o interdependiente.
5. El control: El grado en que se emplean reglas, reglamentos y supervisión directa para vigilar controlar la conducta de los empleados.

6. Tolerancia al riesgo: El grado en que se fomenta que los empleados sean agresivos, innovadores y arriesgados.

7. Los criterios para recompensar: El grado en que se distribuyen las recompensas, como los aumentos de sueldo y los ascensos, de acuerdo con el rendimiento del empleado y no con su antigüedad, favoritismo y otros factores ajenos al rendimiento.

8. Tolerancia al conflicto: El grado en que se fomenta que los empleados traten abiertamente sus conflictos y críticas.

9. El perfil hacia los fines o los medios: El grado en que la administración se perfila hacia los resultados o metas y no hacia las técnicas o procesos usados para alcanzarlos.

10. El enfoque hacia un sistema abierto: El grado en que la organización controla y responde a los cambios del entorno.

La cultura organizativa se constituye a lo largo del tiempo y representa el universo simbólico de la organización. Cada institución cultiva y mantiene su propia cultura y como consecuencia son conocidas por diversas particularidades que los hacen únicos a las demás empresas, lo cual influye en la productividad y en la preocupación por el servicio al cliente (Chiavenato, 2009) De esta manera Trice y Bayer en 1993 mencionaron que "la cultura organizacional se refiere a un sistema de significados que comparten los miembros de una organización y que la distinguen de las demás" (p. 2). Las 
definiciones son diversas, pero es importante concluir que todas las organizaciones tienen una cultura distintiva, sea está favorable o desfavorable para su crecimiento.

Así como se mencionaron diversas definiciones también se crearon modelos sobre este término reciente, planteando la cultura organizacional desde distintos puntos de vista: Modelo de las dimensiones culturales según Hofstede (1980); Modelo de cultura organizacional de Edgar Schein (1988); Modelo sobre las cuestiones ontológicas de las organizaciones de Smircich (1983) y el Modelo cultura organizacional de Denison (1990).

Evidentemente estos tipos de cultura organizacional presentados son diferentes en sus características y en el manejo de la organización. Es así que cada empresa o institución posee sus particularidades y por supuesto que una organización podría tener consigo una mezcla de las características mencionadas, por lo mismo, es importante resaltar que cada uno de los tipos mencionados tiene sus debilidades y sus fortalezas, lo fundamental es que la cultura debe ser consistente y abierta.

La cultura corporativa constituye un factor muy importante para determinar el éxito o el fracaso de las organizaciones. Las organizaciones con éxito adoptan culturas no sólo flexibles, sino, sobre todo, sensibles, para dar cabida a las diferencias sociales y culturales de sus trabajadores, principalmente cuando actúan en términos globales y competitivos, esparciéndose por distintas partes del mundo (Chiavenato, 2009, p. 181).

\section{MÉTODO}

El método se sustentó en el paradigma positivista que según Choque (2014) "se conoce como positivismo a una corriente filosófica que entiende al mundo como independiente del sujeto cognoscente, los resultados científicos tiene un carácter normativo ya que pueden ser generalizados $y$, por ende, ser aplicados a todo tipo de contextos" (p. 15). Se dimensionó bajo el diseño no experimental que de acuerdo con Hernández, Fernández y Baptista (2014) son estudios que se realizan sin la manipulación deliberada de variables y en los que sólo se observan los fenómenos en su ambiente natural para analizarlos. Se empleó el enfoque cuantitativo. Hernández, Fernández, y Baptista (2014) mencionan que este enfoque utiliza la recolección de datos para probar hipótesis con base en la medición numérica y el análisis estadístico, con el fin establecer pautas de comportamiento $y$ probar teorías. El tipo descriptivo propositivo, permitió "especificar propiedades y características importantes de cualquier fenómeno que se analice. Describe tendencias de un grupo o población" (Hernández, Fernández y Baptista, 2014, p. 92). La técnica que se utilizó para la recolección de datos fue la encuesta y cómo instrumento la entrevista semiestructurada. Estimando el Cuestionario de Cultura Organizacional de Denison (1990). La población con la que se trabajó fueron todos los docentes de las cinco facultades de la Universidad Adventista de Bolivia, lo cual hace un total de 125, de ellos respondieron 67 (la muestra) docentes al cuestionario. Para su validez, el instrumento se sometió a un análisis por cuatro juicios de expertos, quienes evaluaron el contenido de cada ítem. 


\section{RESULTADOS}

Los análisis de los resultados fueron obtenidos desde los aspectos antigüedad, religión, género y misión, según los objetivos de investigación: Objetivo General: Caracterizar la percepción de los docentes sobre la cultura organizacional de la Universidad Adventista de Bolivia durante la gestión 1/2020.

\section{Objetivos específicos}

- Describir la implicación de los docentes en las actividades de la Universidad Adventista de Bolivia en la gestión 1/2020.
- Especificar la percepción sobre la consistencia en los docentes de la Universidad Adventista de Bolivia en la gestión 1/2020.

- Identificar la adaptabilidad organizacional de la Universidad Adventista de Bolivia en los docentes de la gestión 1/2020.

En este apartado se muestran los resultados generales de todos los docentes y cómo estos perciben la cultura organizacional, la cual se especifica en las tablas siguientes.

Tabla 1. Cultura organizacional de la UAB

\begin{tabular}{lcc}
\hline Categorías & Fi & $\%$ \\
\hline Débil $(112-163)$ & 2 & 3,0 \\
Estable $(164-213)$ & 15 & 22,4 \\
Fuerte $(214-263)$ & 41 & 61,2 \\
Muy fuerte $(264-310)$ & 9 & 13,4 \\
\hline Total & $\mathbf{6 7}$ & $\mathbf{1 0 0 , 0}$ \\
\hline
\end{tabular}

Fuente: Elaboración propia (2020)

Los resultados revelan que el 3\% percibe una cultura organizacional débil, es decir, existe poca alineación e identificación con los valores de la organización. El control es ejercido a través de procedimientos exhaustivos, por la jerarquía y la burocracia, en donde existe una estructura vertical. Los jefes rigen las normas comportamentales y son impuestas a los niveles bajos sin incentivos sea profesionales o monetarios, como consecuencia no existe un compromiso genuino con la institución. El $61,2 \%$ de los docentes perciben una cultura organizacional fuerte, es decir, los valores y propósitos de la institución, son firmes, coherentes, conocidos y aceptados por los docentes, de ese modo el liderazgo que se ejerce dentro la universidad es reconocida sin imposiciones, lo cual repercute en el éxito institucional gracias a la identificación del trabajador con la organización.

Las dimensiones medidas dentro de la cultura organizacional son percibidas de manera fuerte, pero con falencias en áreas como la implicación, adaptabilidad y misión, las cuales deben reforzarse de manera constante para el logro de objetivos institucionales en el que el trabajador tome un rol activo. 
Tabla 2. Resultados generales por antigüedad

\begin{tabular}{|c|c|c|c|c|c|c|c|c|c|}
\hline & \multirow[t]{2}{*}{ Categorías } & \multicolumn{2}{|c|}{$\begin{array}{c}\text { Medio año a } \\
\text { un año }\end{array}$} & \multicolumn{2}{|c|}{$\begin{array}{l}\text { Un año y } \\
\text { medio a dos } \\
\text { años }\end{array}$} & \multicolumn{2}{|c|}{$\begin{array}{l}\text { Más de dos } \\
\text { años }\end{array}$} & \multicolumn{2}{|c|}{ Total } \\
\hline & & $\mathbf{F i}$ & $\%$ & $\mathrm{Fi}$ & $\%$ & $\mathbf{F i}$ & $\%$ & $\mathbf{F i}$ & $\%$ \\
\hline \multirow{4}{*}{ 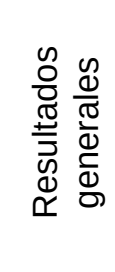 } & Débil (PD. 28 - 39) & 1 & 5 & 1 & 9 & 0 & 0 & 2 & 3 \\
\hline & Estable (PD. 40 - 51 ) & 2 & 9 & 2 & 18 & 11 & 32 & 15 & 22 \\
\hline & Fuerte (PD. 52 - 63) & 15 & 68 & 7 & 64 & 19 & 56 & 41 & 61 \\
\hline & Muy fuerte (PD. 64 - 75) & 4 & 18 & 1 & 9 & 4 & 12 & 9 & 14 \\
\hline Total & & 22 & 100 & 11 & 100 & 34 & 100 & 67 & 100 \\
\hline
\end{tabular}

Fuente: Elaboración propia (2020)

Los docentes con menos de dos años son los que perciben en su mayoría una cultura organizacional fuerte y de ese modo se encuentran mejor alineados a los objetivos. Los docentes con más de dos años de experiencia ya percibieron la cultura de manera más continua y pudieron ver como se trabaja en la estructura, por los mismo el $36 \%$ percibe a la cultura organizacional como estable, la cual contiene limitantes y el docente no se siente satisfecho por el manejo de la misma.

Tabla 3. Resultados generales por religión

\begin{tabular}{|c|c|c|c|c|c|c|c|}
\hline & \multirow{2}{*}{ Categorías } & \multicolumn{2}{|c|}{ Adventista } & \multicolumn{2}{|c|}{ No adventista } & \multicolumn{2}{|c|}{ Total } \\
\hline & & $\mathrm{Fi}$ & $\%$ & $\mathbf{F i}$ & $\%$ & $\mathrm{Fi}$ & $\%$ \\
\hline \multirow{4}{*}{ 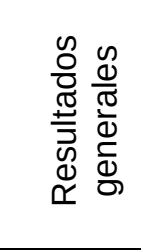 } & Débil (PD. 28 - 39) & 2 & 5 & 0 & 0 & 2 & 3 \\
\hline & Estable (PD. 40 - 51 ) & 13 & 31 & 2 & 8 & 15 & 22 \\
\hline & Fuerte (PD. 52 - 63) & 24 & 57 & 17 & 68 & 41 & 61 \\
\hline & Muy fuerte (PD. 64 - 75) & 3 & 7 & 6 & 24 & 9 & 14 \\
\hline Total & & 42 & 100 & 25 & 100 & 67 & 100 \\
\hline
\end{tabular}

Fuente: Elaboración propia (2020)

Los docentes no adventistas son los que perciben una fuerte cultura organizativa, es decir, se encuentran alineados a este sistema de valores y lo perciben de forma positiva para su desempeño laboral. El estudiante también se siente tomado en cuenta en la organización, por otro lado, en el docente adventista se percibe mayor resistencia a la cultura organizacional, es decir, asumen el manejo de la misma con deficiencias, a pesar que la institución maneja su mismo sistema de valores. 
Tabla 3. Resultados generales por género

\begin{tabular}{|c|c|c|c|c|c|c|c|}
\hline \multicolumn{2}{|r|}{ Categorías } & \multicolumn{2}{|c|}{ Femenino } & \multicolumn{2}{|c|}{ Masculino } & \multicolumn{2}{|c|}{ Total } \\
\hline & & $\mathbf{F i}$ & $\%$ & $\mathbf{F i}$ & $\%$ & $\mathrm{Fi}$ & $\%$ \\
\hline \multirow{4}{*}{ 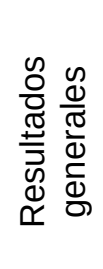 } & Débil (PD. 28 - 39) & 2 & 5 & 0 & 0 & 2 & 3 \\
\hline & Estable (PD. 40 - 51 ) & 11 & 27 & 4 & 15 & 15 & 22 \\
\hline & Fuerte (PD. 52 - 63) & 24 & 58 & 17 & 66 & 41 & 61 \\
\hline & Muy fuerte (PD. 64 - 75) & 4 & 10 & 5 & 19 & 9 & 14 \\
\hline Total & & 41 & 100 & 26 & 100 & 67 & 100 \\
\hline
\end{tabular}

Fuente: Elaboración propia (2020)

Los docentes de género femenino perciben debilidad en la cultura organizacional, es decir, en su mayoría no se sienten identificadas $y$ el conjunto de valores promocionados por la institución queda confuso. El género masculino se encuentra mejor alineado y comprende las estrategias de la organización y se siente más comprometido en el cumplimiento de metas desde su rol docente.

\section{CONCLUSIONES}

Después de exponer los resultados de la investigación realizada con el instrumento del Dr. Denison y la entrevista semiestructurada, en este apartado se presentan las deducciones del trabajo, organizadas según los objetivos específicos planteados inicialmente.

Con respecto a la implicación de los docentes en la Universidad Adventista de Bolivia, se pudo evidenciar que la misma promueve el acto de involucrarse del docente hacia la organización ya que el 43,3\% tiene un fuerte sentido de pertenencia $y$ de responsabilidad hacia la institución. Al margen de aquello también existe un $4,5 \%$ de docentes que no se sienten parte de la institución y que la misma aún tiene falencias en utilizar estrategias que ayuden a que el docente se sienta totalmente parte de la institución y se identifique con ella.
Así mismo la información fue corroborada con la entrevista, en la que el docente menciona que se siente implicado cuando se generan espacios en el que se indaga su opinión y cuándo les brindan herramientas para mejorar sus competencias como profesional, pero cuando esto no se genera, se siente distanciado de la institución.

Con respecto a la percepción sobre la consistencia institucional la conclusión es que el $53,7 \%$ de los docentes perciben la institución con un conjunto de valores claros y que estas son las que direccionan la institución, y la hacen consistente en su estructura, pero también existe un $24 \%$ que percibe que la institución no promueve este conjunto de valores o que simplemente no son difundidas de la manera correcta y que por consecuencia no son aceptadas 0 comprendidas por un cierto grupo.

La información fue corroborada con la entrevista, en la que la consistencia institucional se encuentra fuerte porque el docente se encuentra identificado con la institución bajo diversos factores, del mismo modo con el conjunto de valores que es promocionado por la institución.

Con respecto a identificar la adaptabilidad organizacional la conclusión es que, si bien la universidad procura escuchar al cliente y adaptarse a los cambios, los resultados 
nos muestran que existe falencia en las herramientas de adaptación que utiliza, ya que sólo el $2 \%$ percibe que tiene herramientas de adaptación, pero la mayoría no, y a pesar de que el $67 \%$ perciba una adaptabilidad fuerte, esta se encuentra por debajo de lo ideal. Una de las causas es porque la institución tiene resistencia a nuevos cambios o a escuchar al cliente, es por una estructura vertical, lo cual es necesario trabajar ese aspecto y de ese modo se pueda direccionar como un sistema abierto.

Asimismo, se pudo corroborar con la entrevista, que la institución permite cambios de manera esporádica, es decir, algunas veces, mayormente se percibe una estructura rígida con sus propios lineamientos ya establecidos. De ese modo también con la innovación docente, si bien existen programas y cursos de innovación o apoyo, existen aún lineamientos antiguos que no permiten innovar y crear mejores estrategias.

En cuanto a analizar el conocimiento de la misión la conclusión es que, según el $56,7 \%$ de docentes, la universidad tiene una dirección, propósito y planes claros a corto y a largo plazo, pero existe un $5 \%$ que está totalmente en desacuerdo con el uso de estrategias para alcanzar los objetivos y que además tampoco tienen la misión y visión clara por que la universidad no les brindo los suficientes recursos para identificarse con la organización.

También se puede corroborar con la entrevista que el propósito organizacional no se conoce de manera clara, a causa de que no existe una promoción constante $\mathrm{y}$ significativa que estimule al cumplimiento y a la participación activa del docente dentro del logro de metas y propósitos institucionales.

El objetivo general de la investigación fue caracterizar la percepción de los docentes sobre la cultura organizacional de la Universidad Adventista de Bolivia durante la gestión 1/2020. Se observó durante el análisis como también en las conclusiones generales que la percepción de la población con la que se trabajó, se encuentra dividida en porcentajes considerables. El 61,2\% percibe a la universidad con una cultura fuerte pero que no es el ideal, existe diversas deficiencias que se encuentran evitando que la cultura sea totalmente apropiada por el docente, entonces se puede concluir que si existe una base estable de la cultura organizacional pero que es necesario trabajar con el $25 \%$ de docentes que no opina lo mismo. Para lo cual se recomienda a las autoridades de la Universidad Adventista de Bolivia, proponer jornadas docentes para la ejecución de programas para mejorar la cultura organizacional que serán de beneficio laboral y personal, ya que la implicación también es responsabilidad de cada miembro de la organización.

\section{REFERENCIAS}

Amdeco. (2018). AMDECO - Asociación de Gobiernos Autónomos Municipales de Cochabamba. Recuperado de http://amdeco.org.bo/amdeco/enlacemu nicipios/vinto.php

Chiavenato, I. (2007). Administración de recursos humanos. México: McGrawHill/Interamericana Editores, S.A. DE C.V

Chiavenato, I. (2009). Comportamiento organizacional (2 ed.). Ciudad de México: McGraw-Hill/Interamericana Editores, S.A. de C.V

Chiavenato, I. (2009). Gestión de Talento Humano (3 ed.). Ciudad de México: McGraw-Hill/Interamericana Editores, S.A. DE C.V

Choque, W. (2014). Metodología de la investigación científica. Cochabamba, Bolivia: Universidad Adventista de Bolivia

Denison, D., y Neale, W. (4 de agosto de 1990). Denison Consulting. Recuperado de https://docplayer.net/11003150Denison-organizational-culturesurvey.html 
Hernández, R., Fernández, C., y Baptista, M. d. (2014). Metodología de la investigación (6 ed.). México: McGrawHill / Interamericana Editores, S.A. de C.V

Llanos, M. (2016). La cultura organizacional: eje de acción en la gestión humana. Ecuador: Universidad ECOTEC.

Los Tiempos. (23 de Diciembre de 2016). Los pobladores de Vinto superan los 57 mil. Los Tiempos. Recuperado de https://www.lostiempos.com/actualidad/e conomia/20161223/pobladores-vintosuperan-57-mil

Reina y Valera. (1960). Biblia. Sociedad Biblica en America Latina

Rodríguez, I. (Octubre de 2004). Universidad Estatal a Distancia.
Obtenido de https://www.uned.ac.cr/academica/imag es/igesca/materiales/10.pdf

Schein, E. (1988). La cultura empresarial y el liderazgo. Barcelona: Plaza y Janes Editores, S.A

UAB. (2018). Manual de organización y funciones. Cochabamba: Universidad Adventista de Bolivia

UAB. (2019). Universidad Aventista de Bolivia . Obtenido de https://www.uab.edu.bo/historia-de-lainstitucion/

White, E. G. (1975). La educación cristiana. Buenos Aires: Asociación Casa Editora Sudamericana 